\title{
Evaluation of anidulafungin in the treatment of intra-abdominal candidiasis: a pooled analysis of patient-level data from 5 prospective studies
}

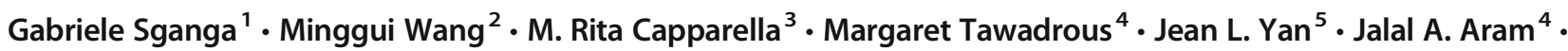 \\ Philippe Montravers ${ }^{6}$
}

Received: 27 February 2019 / Accepted: 14 June 2019 / Published online: 6 July 2019

(C) The Author(s) 2019

\begin{abstract}
The incidence of nosocomial invasive fungal infections involving Candida spp. has increased markedly in recent years in patients undergoing abdominal surgery. This post hoc analysis aimed to determine the efficacy and safety of anidulafungin treatment in patients with intra-abdominal candidiasis (IAC) from five prospective studies (one comparative and four open-label) of adult surgical patients with microbiologically confirmed Candida intra-abdominal infection. Patients received an intravenous (IV) loading dose of anidulafungin $200 \mathrm{mg}$, followed by a daily 100-mg maintenance dose. Per study protocols, some patients could be switched to an oral azole after $\geq 5$ or $\geq 10$ days of IV treatment. Antifungal treatment was maintained for $\geq 14$ days after the last positive Candida culture and resolution of symptoms. The global response rate (GRR) at the end of IV treatment (EOIVT) was the primary endpoint. GRR at the end of therapy (EOT), all-cause mortality at days 14 and 28, and safety was also evaluated. Seventy-nine patients had IAC from peritoneal fluid or hepatobiliary tract. C. albicans $(72.2 \%)$ and C. glabrata (32.9\%) were the most common pathogens. Overall GRR was $73.4 \%$ and $67.1 \%$ at EOIVT and EOT, respectively. All-cause mortality was $17.7 \%$ at day 14 and $24.1 \%$ at day 28 in the modified intent-to-treat population. Anidulafungin was well tolerated in this population, with most adverse events mild or moderate in severity. In these patients with IAC, anidulafungin showed a GRR at EOIVT similar to the anidulafungin registrational trial, and the results of our analysis confirmed the known safety profile of anidulafungin. ClinicalTrials.gov registration number NCT00496197, registered July 3, 2007, https://clinicaltrials.gov/ct2/show/study/ NCT00496197; ClinicalTrials.gov registration number NCT00548262, registered October 19, 2007, https:/clinicaltrials.gov/ ct2/show/record/NCT00548262; ClinicalTrials.gov registration number NCT00537329, registered September 25, 2007, https:// clinicaltrials.gov/ct2/show/record/NCT00537329; ClinicalTrials.gov registration number NCT00689338, registered May 29, 2008, https://clinicaltrials.gov/ct2/show/study/NCT00689338; ClinicalTrials.gov registration number NCT00805740, registered November 26, 2008, https://clinicaltrials.gov/ct2/show/NCT00805740
\end{abstract}

Keywords Anidulafungin $\cdot$ Intra-abdominal candidiasis $\cdot$ Pooled analysis $\cdot$ Patient-level data $\cdot$ Efficacy $\cdot$ Safety

Electronic supplementary material The online version of this article (https://doi.org/10.1007/s10096-019-03617-9) contains supplementary material, which is available to authorized users.

Gabriele Sganga

gabriele.sganga@policlinicogemelli.it

1 Emergency Surgery, Fondazione Policlinico Universitario A. Gemelli IRCCS, Università Cattolica del Sacro Cuore, Largo A. Gemelli 8, 00168 Rome, Italy

2 Huashan Hospital, Fudan University, Shanghai, China
Pfizer PIO, Paris, France

Pfizer Inc, Groton, CT, USA

Pfizer Inc, Collegeville, PA, USA

6 Paris Diderot Sorbonne Cité University and Bichat-Claude Bernard University Hospital, Paris, France 


\section{Introduction}

The incidence of nosocomial invasive fungal infections involving Candida species has increased in recent years because of the increasing number of immunocompromised patients, including patients with cancer, patients undergoing transplants, patients with human immunodeficiency virus and patients in intensive care units (ICUs) [1-4].

Clinical data on intra-abdominal candidiasis (IAC) are still scarce, although IAC is a common type of invasive candidiasis following candidaemia [5]. IAC is confirmed in patients when (i) there is clinical evidence of intra-abdominal infection and (ii) Candida isolates are collected from an intra-abdominal site under sterile conditions within 24 h [5, 6]. IAC, or Candida-associated peritonitis, is a common cause of mortality in patients in the ICU $[1,7,8]$. Previous studies have demonstrated the predominance of C. albicans isolates (approximately 75\%), followed by $C$. glabrata, in intra-abdominal Candida infections in surgical patients $[9,10]$.

High rates of non-albicans Candida isolates from abdominal samples have been reported in patients in the ICU $[7,11]$. In patients admitted to ICUs with post-operative peritonitis, multidrug-resistant strains were frequent and increased with the number of reoperations for persistent abdominal sepsis [12]. In a study of patients with post-operative peritonitis following bariatric surgery, 25 out of 61 patients were positive for Candida infections (6 [22\%] were fluconazole-resistant C. glabrata) and 36 had no Candida infection [13]. Candida infections were isolated more often in late-onset peritonitis and were associated with multidrug-resistant bacteria. Another study demonstrated that $C$. albicans was cultured in 11/18 cases in a bariatric surgery group and in 23/46 cases in a conventional surgery group, whereas $C$. glabrata was cultured in 13/46 cases in the conventional surgery group and 3/6 cases were resistant to fluconazole [14]. A recent, prospective, single-centre, population-based study in a surgical ICU setting observed that IAC was a frequent form of invasive candidiasis (13/22 episodes in 1149 patients) and that antifungal therapy and good source control led to a good outcome. C. albicans and C. parapsilosis were the most common invasive species, and resistance to fluconazole and itraconazole was noted in $3 / 22$ $(13.6 \%)$ cases [15]. To our knowledge, no studies have been published assessing the efficacy of antifungal agents in patients with microbiologically documented IAC.

Among the echinocandin class of antifungal agents, anidulafungin is a cyclic lipopeptide approved for the treatment of candidaemia and other forms of invasive candidiasis such as intra-abdominal abscesses and peritonitis. In the pivotal study of anidulafungin [16], there were only a few patients with microbiologically confirmed Candida deepseated tissue infections. To better understand the efficacy and safety of treatment with anidulafungin in these patients, data from five prospective studies were pooled and analysed.
Here, we present the results of treatment with anidulafungin in the group of patients with microbiologically documented IAC.

\section{Materials and methods}

\section{Ethics}

All primary studies were conducted in compliance with the Declaration of Helsinki and Good Clinical Practice Guidelines established by the International Conference on Harmonization. The final protocols, amendments and informed consent documentation were reviewed and approved by the Institutional Review Boards and the Independent Ethics Committees of the investigational centres. All patients provided written informed consent.

\section{Study design and treatment}

Patient-level efficacy and safety data were retrospectively pooled from four open-label, non-comparative studies [17-20] and one comparative study (Pfizer data on file) with the aim of analysing the safety and efficacy of anidulafungin in the treatment of invasive candidiasis in adult patients. The comparative study was a double-blind, double-dummy, randomised, multicentre trial comparing anidulafungin with caspofungin in patients with microbiologically confirmed deep-seated tissue infection due to Candida species. All the studies had similar protocols and endpoints, and investigated the use of anidulafungin in a broad range of patients, including patients in the ICU and patients with neutropenia (absolute neutrophil count $[\mathrm{ANC}]<500 \mathrm{cells} / \mathrm{mm}^{3}$ ) with microbiologically confirmed Candida intra-abdominal infections (Table 1). All studies were registered with ClinicalTrials.gov (NCT00496197, NCT00548262, NCT00537329, NCT00689338, and NCT00805740).

Patients initiated the treatment with a 200-mg loading dose of intravenous (IV) anidulafungin on day 1 of the study, followed by a maintenance dose of $100 \mathrm{mg}$ IV once daily. In all but one of the studies (A8851022 [NCT00805740]), patients could be switched to an oral azole after $\geq 5$ days (A8851011 [NCT00496197], A8851015 [NCT00548262], A8851016 [NCT00537329]) or $\geq 10$ days (A8851019 [NCT00689338]) of IV treatment, according to pre-specified criteria in each protocol. Antifungal treatment (IV anidulafungin plus subsequent oral azole if used) was maintained for $\geq 14$ days after the last positive Candida culture and resolution of symptoms.

\section{Patients—criteria for the pooled analysis}

The pooled analysis included male or female patients aged $\geq 18$ years with culture-confirmed IAC from a culture specimen obtained within the preceding $96 \mathrm{~h}$ from a normally 
Table 1 Prospective clinical studies included in the pooled analysis (intent-to-treat population ${ }^{\mathrm{a}}$ )

\begin{tabular}{|c|c|c|c|c|}
\hline Study & Region & Type of study & Indication & References \\
\hline A8851011 (NCT00496197) & USA and Korea & Open-label & $\begin{array}{l}\text { Candidaemia and } \\
\text { invasive candidiasis }\end{array}$ & Vazquez et al. 2014 [17] \\
\hline A8851015 (NCT00548262) & Latin America & Open-label & $\begin{array}{l}\text { Candidaemia and } \\
\text { invasive candidiasis }\end{array}$ & Nucci et al. 2014 [18] \\
\hline A8851016 (NCT00537329) ${ }^{\mathrm{b}}$ & Asia & Open-label & Candidaemia & Mootsikapun et al. 2013 [19] \\
\hline A8851019 (NCT00689338) ${ }^{\mathrm{c}}$ & Europe and Canada & Open-label & $\begin{array}{l}\text { Candidaemia and } \\
\text { invasive candidiasis }\end{array}$ & Ruhnke et al. 2012 [20] \\
\hline A8851022 (NCT00805740) & $\begin{array}{l}\text { USA, Canada, Europe, } \\
\text { Russia, Switzerland }\end{array}$ & Double-blind, randomised & $\begin{array}{l}\text { Candida } \text { deep-seated } \\
\text { tissue infection }\end{array}$ & Pfizer data on file \\
\hline
\end{tabular}

${ }^{a}$ All patients who received at least one dose of anidulafungin

${ }^{\mathrm{b}}$ Switch to an oral azole (fluconazole or voriconazole) was permitted after $\geq 5$ days of intravenous treatment ${ }^{\mathrm{c}}$ Switch to an oral azole (fluconazole or voriconazole) was permitted after $\geq 10$ days of intravenous treatment

sterile site or newly placed drain. Patients could enter the studies based on microbiological evidence suggestive of Candida infection (e.g. positive blood culture for yeast). However, confirmation of Candida species was required within $96 \mathrm{~h}$ to remain in the study. Patients were also required to have more than one of the following clinical signs and symptoms of fungal infection: fever, defined as an oral or tympanic temperature $>38{ }^{\circ} \mathrm{C}$; hypotension, defined as systolic blood pressure $<100 \mathrm{mg} \mathrm{Hg}$ or a decrease in systolic blood pressure of $>30 \mathrm{mmHg}$ from baseline; and clinical signs of localised or generalised peritonitis including the presence of intraabdominal abscess or purulent fluid from drains. Patients who had received $>48 \mathrm{~h}$ of prior antifungal therapy had the presence of confirmed or suspected Candida osteomyelitis, endocarditis or meningitis, had prosthetic devices at infection sites that could not be removed within $24 \mathrm{~h}$ of study entry or who had previously failed treatment for the current episode of Candida infection were excluded. From the pooled database, only patients with peritoneal fluid or hepatobiliary tract as sites of infection, as reported by the investigators in the case report form (CRF), were included in this analysis.

\section{Study endpoints}

The primary endpoint of this analysis was the successful global response at the end of IV treatment (EOIVT), based on the modified intent-to-treat (MITT) population. The MITT population included all patients who received $\geq 1$ dose of anidulafungin (intent-to-treat population) and who had a confirmed diagnosis of invasive candidiasis/candidaemia and a positive culture for Candida species within $96 \mathrm{~h}$ of study entry. A global response was considered successful if patients achieved both clinical success (defined as the resolution of signs and symptoms of Candida infection and no additional systemic or oral antifungal therapy required) and microbiological success (defined as eradication or presumed eradication of Candida species present at baseline).
Secondary endpoints of this analysis included the global response at the end of all therapy (EOT) in the MITT population, and the rate of all-cause mortality at day 14 and day 28 after initiation of IV therapy in the MITT populations. Treatment-emergent adverse events (TEAEs) and serious adverse events (SAEs) of all causalities in the MITT population were recorded in all studies and were summarised by Medical Dictionary for Regulatory Activities preferred term.

\section{Statistical analysis}

Efficacy and safety analyses were for descriptive purposes, and no hypotheses for efficacy endpoints were tested. Evaluation of the data comprised primarily of summary descriptive statistics. Success rates for global response were estimated with exact 95\% confidence intervals (CI) for binomial proportion (Clopper-Pearson method). In efficacy analyses, indeterminate or missing was considered failure.

Multivariate logistic regression was used to calculate odds ratios and $95 \% \mathrm{CI}$ to identify factors that were significantly related to treatment failure in the MITT population. Risk factors for developing candidiasis were included in the protocols of all studies. Available patient characteristics data included in the multivariate analysis were age, baseline Acute Physiology and Chronic Health Evaluation (APACHE) II score, body mass index and baseline Candida species (mono- vs polypathogenic infections).

\section{Results}

\section{Patients}

The 5 studies, summarised in Table 1, included 129 anidulafungin-treated patients with deep-seated tissue infection (the MITT population). Of these, 79 patients had microbiologically confirmed intra-abdominal infection with Candida spp. 
isolated from peritoneal fluid or hepatobiliary tract. Baseline characteristics are summarised in Table 2. Neutropenic status was recorded by the investigators for 38 patients only (38/79; $48.1 \%)$. Thirty-five of the 38 patients had an ANC $>500$ cells/ $\mathrm{mm}^{3}(35 / 38 ; 92.1 \%)$ and 3 patients $(7.9 \%)$ had an $\mathrm{ANC} \leq 500$ cells $/ \mathrm{mm}^{3}$. The mean APACHE II score was 15.8 , with 15 patients (19\%) having a score $>20$.

The most frequent baseline pathogens (Table 2) were C. albicans (72.2\%) and C. glabrata (32.9\%), although patients could have more than one pathogen. In vitro minimum inhibitory concentration data for anidulafungin, along with susceptibility to anidulafungin, fluconazole and voriconazole, are shown in Online Resource 1. The peritoneal cavity (70/79; $88.6 \%$ ) was the most common site of infection. Among the nine patients $(9 / 79 ; 11.4 \%)$ with infections in the hepatobiliary tract, two had post-liver transplant complications and seven had biliary infections following liver or biliary tract interventions for malignancies. Ten patients $(10 / 79 ; 12.7 \%)$ also had candidaemia, and three patients had more than one site of infection (peritoneal cavity plus abdomen; peritoneal cavity plus other; peritoneal cavity plus pleural cavity plus other). Eight patients (two hepatobiliary, six peritoneal cavity) had concomitant bacterial infections with bacteria isolated from microbiological culture and reported in the CRF. However, almost all the patients (77/79) were also receiving systemic concomitant antibiotic treatment.

In the MITT population, frequent risk factors for invasive candidiasis included the use of broad-spectrum antibiotics $(85.9 \%)$, surgery $(84.5 \%)$, central venous lines $(76.1 \%)$, and mechanical ventilation $(56.3 \%)$ (Table 2).

The median duration of IV anidulafungin therapy for all patients with IAC was 14.0 days (range 1-42). In the studies where a switch from IV to oral azole therapy was permitted (all except A8851022), the median duration of therapy (IV and oral) was 16.5 days (range 1-56). In total, 26 patients switched to oral azole therapy after a median of 11.5 days (range 6-34); in studies that permitted switch after $\geq 5$ days, 14 patients switched to oral therapy (median 7.0 days [range 5-21]), and in studies that permitted switch after $\geq 10$ days, 12 patients switched (median 11.0 days [range 10-43]).

\section{Outcomes}

In the MITT population, the overall global response rate (GRR) was $73.4 \%$ at EOIVT and $67.1 \%$ at EOT, as shown in Table 3. The GRRs at EOIVT and EOT by most frequent pathogens were $73.7 \%$ and $68.4 \%$ for C. albicans, and $76.9 \%$ and $73.1 \%$ for $C$. glabrata (Table 3 ).

The GRRs at EOIVT and EOT were $77.8 \%$ and $66.7 \%$ for hepatobiliary infections and $73.7 \%$ and $66.7 \%$ for peritoneal cavity infections, respectively. The GRRs at EOIVT in patients with infections caused by a single or multiple Candida isolates were $74.6 \%(50 / 67)$ and $66.7 \%(8 / 12)$, respectively.
Table 2 Patient characteristics at baseline ( $n=79$; MITT population)

\begin{tabular}{|c|c|}
\hline Characteristic & Mean \\
\hline Age, years (SD) & $60.0(16.4)$ \\
\hline Gender (male/female), $n$ & $42 / 37$ \\
\hline \multicolumn{2}{|l|}{ Race, $n(\%)$} \\
\hline $\begin{array}{l}\text { White } \\
\text { Black } \\
\text { Asian } \\
\text { Other/unspecified }\end{array}$ & $\begin{array}{l}65(82.3) \\
4(5.1) \\
1(1.3) \\
9(11.4)\end{array}$ \\
\hline Weight, kg (SD) & $77.3(23.0)$ \\
\hline Height, cm (SD) & $168.1(9.8)$ \\
\hline APACHE II score (SD) & $15.8(6.2)$ \\
\hline \multicolumn{2}{|l|}{ No. of patients (\%) with score: } \\
\hline $\begin{array}{l}\leq 20 \\
>20\end{array}$ & $\begin{array}{l}64(81.0) \\
15(19.0)\end{array}$ \\
\hline \multicolumn{2}{|l|}{ Main baseline pathogen, $n(\%)^{\mathrm{a}}$} \\
\hline $\begin{array}{l}\text { C. albicans } \\
\text { C. glabrata } \\
\text { C. krusei } \\
\text { C. parapsilosis } \\
\text { C. tropicalis } \\
\text { Candida spp. }\end{array}$ & $\begin{array}{l}57(72.2) \\
26(32.9) \\
5(6.3) \\
2(2.5) \\
6(7.6) \\
1(1.3)\end{array}$ \\
\hline \multicolumn{2}{|l|}{ Site of infection, $n(\%)$} \\
\hline $\begin{array}{l}\text { Peritoneal cavity } \\
\text { Peritoneal cavity plus blood } \\
\text { Peritoneal cavity plus other sites } \\
\text { Hepatobiliary }\end{array}$ & $\begin{array}{l}70(88.6) \\
10(12.7) \\
3(3.8) \\
9(11.4)\end{array}$ \\
\hline \multicolumn{2}{|l|}{ Risk factors for invasive candidiasis, $n(\%)$} \\
\hline $\begin{array}{l}\text { Use of broad-spectrum antibiotics } \\
\text { Surgery } \\
\text { Use of central venous catheter } \\
\text { Mechanical ventilation } \\
\text { Length of ICU stay ( }>4 \text { days) } \\
\text { Total parenteral nutrition } \\
\text { Renal insufficiency/failure/dialysis } \\
\text { Use of systemic steroids/immunosuppressives } \\
\text { Solid organ transplant } \\
\text { Chemotherapy } \\
\text { Neutropenia } \\
\text { Other }\end{array}$ & $\begin{array}{l}61(85.9) \\
60(84.5) \\
54(76.1) \\
40(56.3) \\
37(52.1) \\
35(49.3) \\
21(29.6) \\
13(18.3) \\
6(8.5) \\
5(7.0) \\
2(2.8) \\
22(31.0)\end{array}$ \\
\hline
\end{tabular}

ANC absolute neutrophil count, APACHE Acute Physiology and Chronic Health Evaluation, $C R F$ case report form, ICU intensive care unit, MITT modified intent to treat, $S D$ standard deviation

${ }^{a}$ A single patient may have had more than one pathogen; the total percentages therefore add up to $>100 \%$

${ }^{\mathrm{b}}$ Peritoneal cavity plus abdomen; peritoneal cavity plus other; peritoneal cavity plus pleural cavity plus other

${ }^{\mathrm{c}}$ Any surgical intervention (central venous catheter, drainage and abdominal surgery)

${ }^{\mathrm{d}}$ Any severity of renal insufficiency at baseline was included

${ }^{\mathrm{e}}$ Two patients with neutropenia as a risk factor at baseline, as reported by the investigator in the $\mathrm{CRF}$, which did not necessarily correspond to the 3 patients for whom ANC was recorded in the CRF

All-cause mortality at EOIVT and EOT is shown in Table 3. All-cause mortality at day 14 and day 28 was $17.7 \%(14 / 79)$ and 
Table 3 Anidulafungin GRRs (clinical and microbiological) and all-cause mortality (MITT population)

\begin{tabular}{lllll}
\hline Outcome & EOIVT & $95 \%$ CI & EOT & $95 \%$ CI \\
\hline GRR success, $n(\%)$ & $58 / 79(73.4)$ & $63.7-83.2$ & $53 / 79(67.1)$ & $56.7-77.5$ \\
GRR by baseline pathogen ${ }^{\mathrm{a}}, n(\%)$ & & & & \\
C. albicans & $42 / 57(73.7)$ & $62.3-85.1$ & $39 / 57(68.4)$ & $56.4-80.5$ \\
C. glabrata & $20 / 26(76.9)$ & $60.7-93.1$ & $19 / 26(73.1)$ & $56.0-90.1$ \\
C. krusei & $4 / 5(80.0)$ & $44.9-100.0$ & $4 / 5(80.0)$ & $44.9-100.0$ \\
C. parapsilosis & $1 / 2(50.0)$ & $0.0-100.0$ & $1 / 2(50.0)$ & $0.0-100.0$ \\
C. tropicalis & $3 / 6(50.0)$ & $10.0-90.0$ & $2 / 6(33.3)$ & $0.0-71.1$ \\
Candida spp. & $1 / 1(100.0)$ & & $1 / 1(100.0)$ & \\
$\quad$ Candida (unspecified) & $1 / 1(100)$ & & $1 / 1(100)$ & \\
GRR by site of infection, $n(\%)$ & & & & \\
Hepatobiliary & $7 / 9(77.8)$ & $50.6-100.0$ & $6 / 9(66.7)$ & $35.9-97.5$ \\
Peritoneal cavity & $42 / 57(73.7)$ & $62.3-85.1$ & $38 / 57(66.7)$ & $54.4-78.9$ \\
Peritoneal cavity plus blood & $7 / 10(70.0)$ & $41.6-98.4$ & $7 / 10(70.0)$ & $41.6-98.4$ \\
Peritoneal cavity plus other sites ${ }^{\mathrm{b}}$ & $2 / 3(66.7)$ & $13.3-100.0$ & $2 / 3(66.7)$ & $13.3-100.0$ \\
All-cause mortality (no. of deaths) & $5 / 79(6.3)$ & & $6 / 79(7.6)$ & \\
\hline
\end{tabular}

$C I$ confidence interval, EOT end of therapy, EOIVT end of intravenous therapy, GRR global response rate; MITT modified intent to treat

${ }^{\text {a }}$ Main pathogens at baseline

${ }^{\mathrm{b}}$ Peritoneal cavity plus abdomen; peritoneal cavity plus other; peritoneal cavity plus pleural cavity plus other
$24.1 \%$ (19/79), respectively, in the MITT population. Due to limited data for neutropenia, sub-analysis was not possible.

Multivariate logistic regression was used to identify factors that were significantly related to failure. For the analysis, age, body mass index, baseline APACHE II score and baseline Candida species (infections caused by single vs multiple Candida species) were evaluated. None of the assessed factors were associated with failure.

\section{Safety and tolerability}

The incidence and severity of TEAEs during anidulafungin IV treatment are shown in Table 4. Anidulafungin was well tolerated in this population, and most adverse events were mild or moderate in severity. SAEs were noted in 39 patients $(49.4 \%)$, with the most frequent being septic shock $(n=8)$, multipleorgan dysfunction syndrome $(n=3)$, cardiac arrest $(n=3)$, hypotension $(n=3)$ and haemorrhagic shock $(n=3)$.

\section{Discussion}

To our knowledge, this study represents the largest collection of patients treated with anidulafungin in microbiologically documented intra-abdominal infections in a clinical trial setting. The pooled analysis demonstrates that in the population of surgical patients with IAC treated with anidulafungin, the GRR was similar to that in the anidulafungin registrational trial [16]. The low incidence of candidaemia $(12.7 \%)$ was
Table 4 Incidence and severity of TEAEs by system organ class during intravenous treatment with anidulafungin (MedDRA preferred terms $>2 \%$ by system organ class)

\begin{tabular}{lllll}
\hline Category & $n(\%)$ & Mild & Moderate & Severe \\
\hline Any AE & $11(13.9)$ & 8 & 3 & 0 \\
Blood and lymphatic system & $2(2.5)$ & 1 & 1 & 0 \\
Anaemia & $1(1.3)$ & 1 & 0 & 0 \\
Thrombocytopenia & $1(1.3)$ & 0 & 1 & 0 \\
Cardiac & $2(2.5)$ & 2 & 0 & 0 \\
Cardiac failure congestive & $1(1.3)$ & 1 & 0 & 0 \\
Myocardial ischaemia & $1(1.3)$ & 1 & 0 & 0 \\
Supraventricular tachycardia & $1(1.3)$ & 1 & 0 & 0 \\
Tachycardia & $1(1.3)$ & 1 & 0 & 0 \\
Gastrointestinal & $2(2.5)$ & 2 & 0 & 0 \\
Abdominal pain & $1(1.3)$ & 1 & 0 & 0 \\
Diarrhoea & $1(1.3)$ & 1 & 0 & 0 \\
Investigations & $5(6.3)$ & 4 & 1 & 0 \\
Aspartate aminotransferase $\uparrow$ & $1(1.3)$ & 1 & 0 & 0 \\
Blood alkaline phosphatase $\uparrow$ & $3(3.8)$ & 3 & 0 & 0 \\
Immunosuppressant drug level $\uparrow$ & $1(1.3)$ & 0 & 1 & 0 \\
Nervous system & $2(2.5)$ & 2 & 0 & 0 \\
Headache & $1(1.3)$ & 1 & 0 & 0 \\
Paraesthesia & $1(1.3)$ & 1 & 0 & 0 \\
Skin and subcutaneous tissue & $2(2.5)$ & 2 & 0 & 0 \\
Pruritus & $1(1.3)$ & 1 & 0 & 0 \\
Rash & $1(1.3)$ & 1 & 0 & 0 \\
Vascular & $2(2.5)$ & 1 & 1 & 0 \\
Hypertension & $1(1.3)$ & 1 & 0 & 0 \\
Hypotension & $1(1.3)$ & 0 & 1 & 0 \\
\hline
\end{tabular}

$A E$ adverse event, MedDRA Medical Dictionary for Regulatory Activities, TEAE treatment-emergent adverse event 
comparable with that in a recent epidemiological study [5]. Our findings are also consistent with other investigations [5, 9], in terms of C. albicans (72.2\%) and C. glabrata (32.9\%) being the most common pathogens causing IAC at baseline. However, C. parapsilosis was not as common in this study (only 2 isolates at baseline), despite being one of the most common pathogens in another investigation (22.7\%) [15]. In this pooled analysis, all Candida species were susceptible to anidulafungin, $84.2 \%$ were susceptible to fluconazole and $94.7 \%$ were susceptible to voriconazole. The superior susceptibility of anidulafungin over fluconazole was consistent with a study in a murine model of candidiasis [21].

Our study confirmed that abdominal surgery and use of broad-spectrum antibiotics are the most important risk factors associated with intra-abdominal invasive candidiasis.

Interestingly, in this study only 8 out of 79 patients had bacteria isolated from microbiological culture and recorded in the CRF. However, almost all patients (77/79) were receiving systemic antibiotic treatments, indicating concomitant bacterial infections, although it was not possible to know more about the sites of bacterial infection as this information was not collected in the CRF.

The median duration of IV anidulafungin in patients with intra-abdominal infections was 14 days, which was longer than the 10 days of IV anidulafungin duration described in the pooled analysis of the overall general population [22]. In the real-world AmarCAND2 study, the median duration of antifungal therapy was 14 days for targeted therapy and 17 days for empirical therapy [11]. Furthermore, in our population, a lower percentage of the patients (22\% and $19 \%$; data not shown) switched to oral therapy compared to that found by Kullberg et al. [22], where $51.8 \%$ and $34.8 \%$ of patients switched when permitted after $\geq 5$ or $\geq 10$ days, respectively.

Across the five studies, anidulafungin treatment was well tolerated, with most TEAEs mild or moderate in severity, consistent with the known safety profile of anidulafungin demonstrated since the registrational trials.

The anidulafungin registration trial included few patients with IAC. The pooled analysis of anidulafungin studies included 129 patients with deep-seated tissue infection [22], of whom 79 in the present study had confirmed IAC from peritoneal fluid and hepatobiliary tract. In general, other studies with echinocandins have included low numbers of patients with IAC. In one investigation where 16 patients had Candida peritonitis, the success rate was $87.5 \%$ (7/8) with amphotericin B and 100\% (8/8) for caspofungin [23]. Success rates of $72.7 \%$ with liposomal amphotericin B and $82.4 \%$ with micafungin were observed in 28 patients with invasive candidiasis in the peritoneum [24]. In a study of 18 patients with Candida peritonitis, a $40 \%$ success rate was seen with caspofungin compared with $66.7 \%$ and $57.1 \%$ with micafungin $100 \mathrm{mg}$ and $150 \mathrm{mg}$, respectively [25]. In a prospective study of 279 adult patients in the ICU, C. albicans was involved in $67 \%$ and C. glabrata in $15.6 \%$ [11]. Of the 179 Candida strains cultured from peritoneal samples, C. albicans was cultured in 61/96 (64\%) isolates in empirically treated patients and in 59/83 (71\%) receiving targeted therapy. Of the 179 empiric and targeted patients, 3 had candidaemia. Risk factors that influenced a higher mortality in the AmarCAND2 study included healthcare-associated Candida peritonitis, Sequential Organ Failure Assessment score $\geq 8$ at ICU admission and Simplified Acute Physiologic Score II $\geq 45$ at systemic antifungal treatment initiation [11]. Findings from the current analysis are similar but with lower mortality.

Intra-abdominal candidiasis is still poorly understood compared with candidaemia. To date, data and studies on the efficacy of echinocandins in intra-abdominal invasive candidiasis are scarce and, although IAC is still burdened by high mortality rates, all current international guidelines mainly address candidaemia. Source control is of paramount importance in the treatment of IAC, but adequate antifungal therapy is essential to eradicate the pathogen and to treat candidaemia, when present. For this reason, guidelines recommend an echinocandin as the drug of choice [26-28], although the Infectious Diseases Society of America does not include guidance on duration of therapy [26].

The concept of early adequate systemic antifungal therapy in patients with IAC is a point of debate based on the assumption that delayed initiation of antifungal therapy is associated with a poor outcome, as reported for patients with IAC, particularly if they also have candidaemia [29-33]. The deleterious impact of delayed initiation of systemic antifungal treatment has not yet been demonstrated for Candida intraabdominal infection [5], and further evidence is needed.

A limitation of the current investigation was that four studies were open-label and were conducted in different centres within different timeframes, and yielded a small number of patients. In mitigation, all studies had similar protocols and endpoints that allowed the data to be pooled. Although two pharmacokinetic studies have demonstrated adequate anidulafungin plasma concentrations [34, 35], the lack of data to demonstrate that anidulafungin achieves adequate penetration of the peritoneal cavity may also be considered a limitation of this analysis. The analysis did not permit the assessment of whether anidulafungin would behave differently from other echinocandins in this population. Furthermore, this post hoc analysis did not include a control group and was not preplanned by the protocols of the individual studies. It was, therefore, not possible to evaluate the adequacy of source control or of antibacterial therapy. In addition, assessment of global response and mortality in relation to the neutropenic status of patients could not be performed because data on neutropenic status were not available for all patients. Finally, only a small number of factors (age, body mass index, baseline APACHE II score and baseline Candida species) were 
evaluated by multivariate logistic regression to assess any association with failure, and none of these factors were found to be associated with failure.

This analysis of pooled data showed that the epidemiology of IAC is similar to that in previous studies, with C. albicans and C. glabrata being the most common pathogens. Also, IAC seemed to be accompanied by a lower incidence of candidaemia compared with other studies [5, 36]. Anidulafungin provided good efficacy and tolerability, which is an important consideration in critically ill surgical patients with IAC in the ICU.

Nevertheless, further studies are needed to evaluate the clinical efficacy of echinocandins, as well as their tissue penetration into the peritoneum and possibly in specific intra-abdominal districts such as bile, the liver and the pancreas.

Acknowledgements Data from this analysis were presented at the 55th Interscience Conference of Antimicrobial Agents and Chemotherapy (ICAAC) meeting, San Diego, CA, USA, 17-21 September 2015. Editorial/medical writing support, under the direction of the authors, was provided by Neil Cockburn of CMC Connect, a division of McCann Health Medical Communications Ltd., Macclesfield, UK, funded by Pfizer, in accordance with Good Publication Practice (GPP3) guidelines (Ann Intern Med 2015;163:461-4).

Research data sharing Upon request, and subject to certain criteria, conditions and exceptions (see https://www.pfizer.com/science/clinicaltrials/trial-data-and-results for more information), Pfizer will provide access to individual de-identified participant data from Pfizer-sponsored global interventional clinical studies conducted for medicines, vaccines and medical devices (1) for indications that have been approved in the US and/or EU or (2) in programmes that have been terminated (i.e. development for all indications has been discontinued). Pfizer will also consider requests for the protocol, data dictionary and statistical analysis plan. Data may be requested from Pfizer trials 24 months after study completion. The de-identified participant data will be made available to researchers whose proposals meet the research criteria and other conditions, and for which an exception does not apply, via a secure portal. To gain access, data requestors must enter into a data access agreement with Pfizer.

Funding This work was supported by Pfizer. Medical writing support was funded by Pfizer.

\section{Compliance with ethical standards}

Conflict of interest G. Sganga is an employee of Catholic University of the Sacred Heart, Fondazione Agostino Gemelli University Hospital, Rome, Italy. M. Wang is an employee of Huashan Hospital, Fudan University, Shanghai, China. M.R. Capparella is an employee and shareholder of Pfizer PIO, Paris, France. M. Tawadrous, J.L. Yan and J.A. Aram are employees and shareholders of Pfizer Inc, USA. P. Montravers has no conflict of interest to declare.

Ethical approval All primary studies were conducted in compliance with the Declaration of Helsinki and Good Clinical Practice Guidelines established by the International Conference on Harmonization. The final protocols, amendments and informed consent documentation were reviewed and approved by the Institutional Review Boards and the Independent Ethics Committees of the investigational centres.
Informed consent All patients provided written informed consent.

Open Access This article is distributed under the terms of the Creative Commons Attribution 4.0 International License (http:// creativecommons.org/licenses/by/4.0/), which permits unrestricted use, distribution, and reproduction in any medium, provided you give appropriate credit to the original author(s) and the source, provide a link to the Creative Commons license, and indicate if changes were made.

\section{References}

1. Delaloye J, Calandra T (2014) Invasive candidiasis as a cause of sepsis in the critically ill patient. Virulence 5:161-169. https://doi. org/10.4161/viru.26187

2. Falagas ME, Roussos N, Vardakas KZ (2010) Relative frequency of albicans and the various non-albicans Candida spp among candidemia isolates from inpatients in various parts of the world: a systematic review. Int J Infect Dis 14:e954-e966

3. Horn DL, Neofytos D, Anaissie EJ, Fishman JA, Steinbach WJ, Olyaei AJ, Marr KA, Pfaller MA, Chang CH, Webster KM (2009) Epidemiology and outcomes of candidemia in 2019 patients: data from the prospective antifungal therapy alliance registry. Clin Infect Dis 48:1695-1703

4. Rabin AS, Givertz MM, Couper GS, Shea MM, Peixoto D, Yokoe DS, Baden LR, Marty FM, Koo S (2015) Risk factors for invasive fungal disease in heart transplant recipients. J Heart Lung Transplant 34:227-232

5. Bassetti M, Righi E, Ansaldi F, Merelli M, Scarparo C, Antonelli M, Garnacho-Montero J, Diaz-Martin A, Palacios-Garcia I, Luzzati R, Rosin C, Lagunes L, Rello J, Almirante B, Scotton PG, Baldin G, Dimopoulos G, Nucci M, Munoz P, Vena A, Bouza E, de Egea V, Colombo AL, Tascini C, Menichetti F, Tagliaferri E, Brugnaro P, Sanguinetti M, Mesini A, Sganga G, Viscoli C, Tumbarello M (2015) A multicenter multinational study of abdominal candidiasis: epidemiology, outcomes and predictors of mortality. Intensive Care Med 41:1601-1610. https://doi.org/10.1007/s00134-015-3866-2

6. Vergidis P, Clancy CJ, Shields RK, Park SY, Wildfeuer BN, Simmons RL, Nguyen MH (2016) Intra-abdominal candidiasis: the importance of early source control and antifungal treatment. PLoS One 11:e0153247

7. Montravers P, Mira J-P, Gangneux J-P, Leroy O, Lortholary O (2011) A multicentre study of antifungal strategies and outcome of Candida spp. peritonitis in intensive-care units. Clin Microbiol Infect 17:1061-1067

8. Hasibeder W, Halabi M (2014) Candida peritonitis. Minerva Anestesiol 80:470-481

9. Dupont H, Paugam-Burtz C, Muller-Serieys C, Fierobe L, Chosidow D, Marmuse JP, Mantz J, Desmonts JM (2002) Predictive factors of mortality due to polymicrobial peritonitis with Candida isolation in peritoneal fluid in critically ill patients. Arch Surg 137:1341-1346. https://doi.org/10.1001/archsurg.137.12. 1341

10. Sandven P, Qvist H, Skovlund E, Giercksky KE (2002) Significance of Candida recovered from intraoperative specimens in patients with intra-abdominal perforations. Crit Care Med 30: 541-547

11. Montravers P, Perrigault PF, Timsit JF, Mira JP, Lortholary O, Leroy O, Gangneux JP, Guillemot D, Bensoussan C, Bailly S, Azoulay E, Constantin JM, Dupont H (2017) Antifungal therapy for patients with proven or suspected Candida peritonitis: Amarcand2, a prospective cohort study in French intensive care units. Clin Microbiol Infect 23:117.e111-117.e8 
12. Montravers P, Dufour G, Guglielminotti J, Desmard M, Muller C, Houissa H, Allou N, Marmuse JP, Augustin P (2015) Dynamic changes of microbial flora and therapeutic consequences in persistent peritonitis. Crit Care 19:70

13. Zappella N, Desmard M, Chochillon C, Ribeiro-Parenti L, Houze S, Marmuse JP, Montravers P (2015) Positive peritoneal fluid fungal cultures in postoperative peritonitis after bariatric surgery. Clin Microbiol Infect 21:853.e851-853.e853

14. Montravers P, Guglielminotti J, Zappella N, Desmard M, Muller C, Fournier P, Marmuse JP, Dufour G, Augustin P (2013) Clinical features and outcome of postoperative peritonitis following bariatric surgery. Obes Surg 23:1536-1544

15. Aguilar G, Delgado C, Corrales I, Izquierdo A, Gracia E, Moreno T, Romero E, Ferrando C, Carbonell JA, Borrás R, Navarro D, Belda FJ (2015) Epidemiology of invasive candidiasis in a surgical intensive care unit: an observational study. BMC Res Notes 8:491. https://doi.org/10.1186/s13104-015-1458-4

16. Reboli AC, Rotstein C, Pappas PG, Chapman SW, Kett DH, Kumar D, Betts R, Wible M, Goldstein BP, Schranz J, Krause DS, Walsh TJ (2007) Anidulafungin versus fluconazole for invasive candidiasis. N Engl J Med 356:2472-2482

17. Vazquez J, Reboli AC, Pappas PG, Patterson TF, Reinhardt J, ChinHong P, Tobin E, Kett DH, Biswas P, Swanson R (2014) Evaluation of an early step-down strategy from intravenous anidulafungin to oral azole therapy for the treatment of candidemia and other forms of invasive candidiasis: results from an open-label trial. BMC Infect Dis 14:97

18. Nucci M, Colombo AL, Petti M, Magana M, Abreu P, Schlamm HT, Sanchez SP (2014) An open-label study of anidulafungin for the treatment of candidaemia/invasive candidiasis in Latin America. Mycoses 57:12-18

19. Mootsikapun P, Hsueh PR, Talwar D, Co VM, Rajadhyaksha V, Ong ML (2013) Intravenous anidulafungin followed optionally by oral voriconazole for the treatment of candidemia in Asian patients: results from an open-label phase III trial. BMC Infect Dis 13:219

20. Ruhnke M, Paiva JA, Meersseman W, Pachl J, Grigoras I, Sganga G, Menichetti F, Montravers P, Auzinger G, Dimopoulos G, Borges Sá M, Miller PJ, Marcek T, Kantecki M (2012) Anidulafungin for the treatment of candidaemia/invasive candidiasis in selected critically ill patients. Clin Microbiol Infect 18:680-687

21. Gumbo T, Drusano GL, Liu W, Ma L, Deziel MR, Drusano MF, Louie A (2006) Anidulafungin pharmacokinetics and microbial response in neutropenic mice with disseminated candidiasis. Antimicrob Agents Chemother 50:3695-3700. https://doi.org/10. 1128/AAC.00507-06

22. Kullberg BJ, Vasquez J, Mootsikapun P, Nucci M, Paiva JA, Garbino J, Yan JL, Aram J, Capparella MR, Conte U, Schlamm H, Swanson R, Herbrecht R (2017) Efficacy of anidulafungin in 539 patients with invasive candidiasis: a patient-level pooled analysis of six clinical trials. J Antimicrob Chemother 72:2368-2377

23. Mora-Duarte J, Betts R, Rotstein C, Colombo AL, ThompsonMoya L, Smietana J, Lupinacci R, Sable C, Kartsonis N, Perfect J, Caspofungin Invasive Candidiasis Study Group (2002) Comparison of caspofungin and amphotericin B for invasive candidiasis. N Engl J Med 347:2020-2029

24. Kuse ER, Chetchotisakd P, da Cunha CA, Ruhnke M, Barrios C, Raghunadharao D, Sekhon JS, Freire A, Ramasubramanian V, Demeyer I, Nucci M, Leelarasamee A, Jacobs F, Decruyenaere J, Pittet D, Ullmann AJ, Ostrosky-Zeichner L, Lortholary O, Koblinger S, Diekmann-Berndt H, Cornely OA (2007) Micafungin versus liposomal amphotericin B for candidaemia and invasive candidosis: a phase III randomised double-blind trial. Lancet 369:1519-1527
25. Pappas PG, Rotstein CM, Betts RF, Nucci M, Talwar D, De Waele JJ, Vazquez JA, Dupont BF, Horn DL, Ostrosky-Zeichner L, Reboli AC, Suh B, Digumarti R, Wu C, Kovanda LL, Arnold LJ, Buell DN (2007) Micafungin versus caspofungin for treatment of candidemia and other forms of invasive candidiasis. Clin Infect Dis 45:883-893

26. Pappas PG, Kauffman CA, Andes DR, Clancy CJ, Marr KA, Ostrosky-Zeichner L, Reboli AC, Schuster MG, Vazquez JA, Walsh TJ, Zaoutis TE, Sobel JD (2016) Clinical practice guideline for the management of candidiasis: 2016 update by the Infectious Diseases Society of America. Clin Infect Dis 62:e1-e50

27. Cornely OA, Bassetti M, Calandra T, Garbino J, Kullberg BJ, Lortholary O, Meersseman W, Akova M, Arendrup MC, ArikanAkdagli S, Bille J, Castagnola E, Cuenca-Estrella M, Donnelly JP, Groll AH, Herbrecht R, Hope WW, Jensen HE, Lass-Flörl C, Petrikkos G, Richardson MD, Roilides E, Verweij PE, Viscoli C, Ullmann AJ (2012) ESCMID* guideline for the diagnosis and management of Candida diseases 2012: non-neutropenic adult patients. Clin Microbiol Infect 18:19-37. https://doi.org/10.1111/ 1469-0691.12039

28. Tissot F, Agrawal S, Pagano L, Petrikkos G, Groll AH, Skiada A, Lass-Flörl C, Calandra T, Viscoli C, Herbrecht R (2017) ECIL-6 guidelines for the treatment of invasive candidiasis, aspergillosis and mucormycosis in leukemia and hematopoietic stem cell transplant patients. Haematologica 102:433-444

29. Garey KW, Rege M, Pai MP, Mingo DE, Suda KJ, Turpin RS, Bearden DT (2006) Time to initiation of fluconazole therapy impacts mortality in patients with candidemia: a multi-institutional study. Clin Infect Dis 43:25-31. https://doi.org/10.1086/504810

30. Kollef M, Micek S, Hampton N, Doherty JA, Kumar A (2012) Septic shock attributed to Candida infection: importance of empiric therapy and source control. Clin Infect Dis 54:1739-1746

31. Lortholary O, Renaudat C, Sitbon K, Madec Y, Denoeud-Ndam L, Wolff M, Fontanet A, Bretagne S, Dromer F (2014) Worrisome trends in incidence and mortality of candidemia in intensive care units (Paris area, 2002-2010). Intensive Care Med 40:1303-1312

32. Morrell M, Fraser VJ, Kollef MH (2005) Delaying the empiric treatment of candida bloodstream infection until positive blood culture results are obtained: a potential risk factor for hospital mortality. Antimicrob Agents Chemother 49:3640-3645

33. Shan YS, Sy ED, Wang ST, Lee JC, Lin PW (2006) Early presumptive therapy with fluconazole for occult Candida infection after gastrointestinal surgery. World J Surg 30:119-126

34. Brüggemann RJ, Middel-Baars V, de Lange DW, Colbers A, Girbes AR, Pickkers P, Swart EL (2017) Pharmacokinetics of anidulafungin in critically ill intensive care unit patients with suspected or proven invasive fungal infections. Antimicrob Agents Chemother 61:e01894-e01816. https://doi.org/10.1128/ AAC.01894-16

35. Dupont H, Massias L, Jung B, Ammenouche N, Montravers $\mathrm{P}$ (2017) Pharmacokinetic study of anidulafungin in ICU patients with intra-abdominal candidiasis. J Antimicrob Chemother 72: 1429-1432. https://doi.org/10.1093/jac/dkw568

36. Maseda E, Rodríguez-Manzaneque M, Dominguez D, GonzálezSerrano M, Mouriz L, Álvarez-Escudero J, Ojeda N, SánchezZamora P, Granizo JJ, Giménez MJ (2016) Intraabdominal candidiasis in surgical ICU patients treated with anidulafungin: a multicenter retrospective study. Rev Esp Quimioter 29:32-39

Publisher's note Springer Nature remains neutral with regard to jurisdictional claims in published maps and institutional affiliations. 